\title{
DERIVATIVES OF 4-GLUCOSIDO-MANNOSE
}

\author{
By Horace S. Issell
}

\section{ABSTRACT}

The reactions of hepta-acetyl-4-glucosido- $\gamma$-methyl-mannoside are considered in detail. It is shown that one acetyl group in hepta-acetyl-4-glucosido- $\gamma$-methylmannoside is resistant to alkaline hydrolysis and that it reacts with hydrogen chloride in chloroform solution to give a chloroacetyl derivative. This reaction explains the formation of the $\beta$-acetyl-methyl-glycoside which was found when the substance was treated, in dry methyl alcohol, successively with hydrogen chloride and silver carbonate. On the other hand, if moist silver carbonate is used in place of the dry salt a hexa-acetyl-4-glucosido-mannose is obtained. These reactions are indicative of the ortho ester structure.

The optical rotations of the derivatives of 4-glucosido-mannose are correlated with the rotations of the analogous derivatives of mannose. The rotations of certain derivatives of 4-glucosido-mannose corresponding to the derivatives of mannose which give exceptional rotations are likewise cxceptional in respect to the numerical relations which are obtained from other sugars. Since 4-glucosidomannose can not form a 1, 4 ring, thesc exceptional rotations are not caused by the existence of that structure.

\section{CONTENTS}

I. Introduction

II. Reactions of hepta-acetyl-4-glucosido- $\gamma$-methyl-mannoside _...... 1117

III. Correlation of the derivatives of 4-glucosido-mannose with the derivatives of mannose . . . . . . .

IV. Experimental details_._._.

1. Hepta-acetyl-4-glucosido- $\gamma$-methyl-mannoside

2. Hepta-acetyl-4-glucosido- $\beta$-methyl-mannoside_........... 1125

3. 4-glucosido- $\beta$-methyl-mannoside_._. $\ldots \ldots \ldots \ldots$

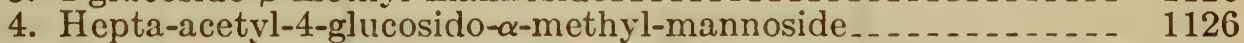

5. Hexa-acetyl-4-glucosido-mannose ........................ 1127

6. Preparation of chloro-hepta-acctyl-4-glucosido-mannose from hepta-acetyl-4-glucosido- $\gamma$-methyl-mannoside........... 1128

7. Hepta-acetyl-4-glucosido-manuose $\ldots \ldots \ldots$

8. 4-glucosido- $\beta$-mannose

9. $\beta$-octa-acetyl-4-glucosido-mannose $\ldots \ldots \ldots \ldots$

V. Acknowledgment_.............. 1131

\section{INTRODUCTIOIN}

In previous publications ${ }^{1}$ it was shown that the molecular rotations of 4-glucosido- $\alpha$-mannose and 4-glucosido- $\beta$-mannose parallel the rotations of $\alpha$ - and $\beta$-mannose. The conclusions as to the structure of $\alpha$ - and $\beta$-mannose drawn from these observations were in opposition to the conclusions previously drawn by C. S. Hudson ${ }^{2}$ from the molecular rotations of the halogen acetyl derivatives of the same sugars. These conflicting views required the extension of the comparison to include a considerable number of mannose derivatives rather than a few selected compounds.

In 1920 Fischer, Bergmann, and Rabe ${ }^{3}$ found that three isomeric acetyl methyl rhamnosides are formed by the interaction of bromo-

1 Isbell, B. S. Jour. Research, vol. 5 (RP253), p. 1179; 1930; also Proc. National Academy of Sciences, vol. 16, p. $704 ; 1930$.

Fudson, J. Am. Chem. Soc., vol. 52, p. 1698; 1930.

Fischer, Bergmann, and Rabe, Ber., vol. 53, p. 2362; 1920. 
acetyl rhamnose, methyl alcohol, and silver carbonate. Subsequently J. K. Dale ${ }^{4}$ reported a series of closely related mannose compounds.

While recently the writer ${ }^{5}$ reported the preparation of three isomeric hepta-acetyl-4-glucosido-methyl-mannosides from bromohepta-acetyl-4-glucosido-mannose by the Koenigs and Knorr reaction, ${ }^{6}$ Haworth, Hirst, Streight, Thomas, and Webb ${ }^{7}$ have also recently reported the preparation of hepta-acetyl-4-glucosido- $\alpha$-methylmannoside. Consequently the writer has devoted most of his attention to the beta and gamma isomers which Haworth and his coworkers were not successful in obtaining in the crystalline state. The three new acetyl derivatives of 4-glucosido-mannose are analogous to the acetyl methyl rhamnosides and mannosides. In accordance with the nomenclature previously used for the analogous derivatives of mannose the isomer $\left(\left[\alpha\left[_{D}^{20}+=29.3\right.\right.\right.$; which gives the highest dextro rota-

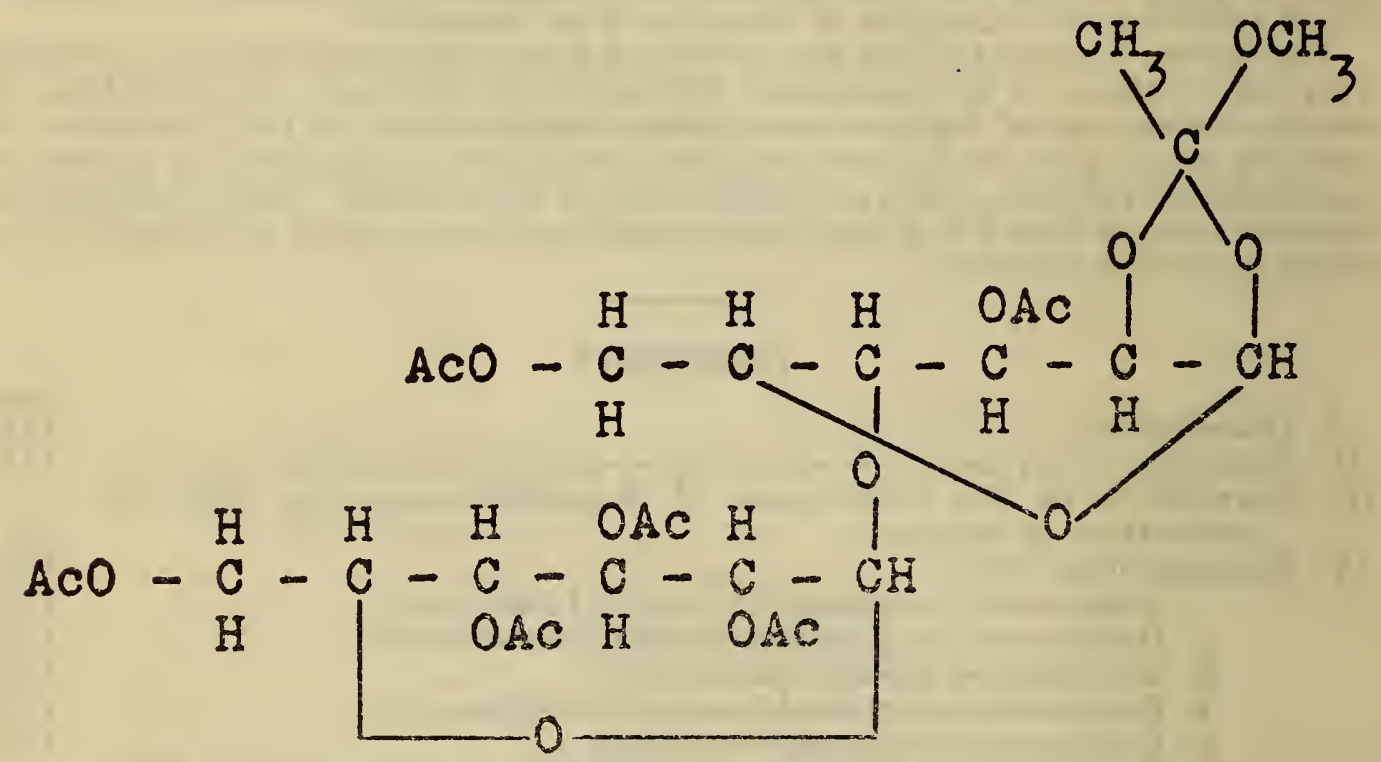

Hepta-acetyl-4-glucosido- $\gamma$-methyl-mannoside

tion is designated alpha; the substance $\left([\alpha]_{D}^{20}=-23.2\right)$ which gives the most negative rotation is designated beta; and the compound which gives the reactions characteristic of the third variety of the acetyl methyl mannosides is designated as the gamma acetyl methyl glycoside.

The gamma or third variety of the acetylated methyl rhamnoside, as well as the corresponding mannose and lyxose ${ }^{8}$ derivatives, exhibits certain peculiar properties which led Fischer, Bergmann, and Rabe to believe that it might have a 1, 3 ring structure. However, recent methylation studies by Haworth, Hirst, and Miller ${ }^{9}$ indicate that all three of the crystalline acetyl methyl rhamnosides have the 1, 5 ring. In order to explain the anomaly of three isomers of the same ring structure, they postulated a new type of isomerism. Shortly thereafter K. Freudenberg ${ }^{10}$ suggested the ortho ester struc-

4 Dale, J. Am. Chem. Soc., vol. 46, p. 1046; 1924

5 Isbell, J. Am. Chem. Soc., vol. 52, p. 5298; 1930.

6 Koenigs and Knorr, Ber., vol. 34, p. 957 ; 1901.

7 Haworth, Hirst, Streight, Thomas, and Webb, J. Chem. Soc., p. 2636; 1930.

8 Levene and Wolfrom, J. Biol. Chem., vol. 79, p. 471; 1928.

- Haworth, Hirst, and Miller, J. Chem. Soc., p. 2469; 1929.

10 Freudenberg, Naturwissenschaften, vol. 18, p. 393; 1930. 
ture and about the same time Bott, Haworth, and Hirst ${ }^{11}$ independently arrived at the same explanation of the new type of isomerism. The ortho ester structure of hepta-acetyl-4-glucosido-mannose is given on page 1116. This structure accounts for the interesting properties of the substance which are considered in the next paragraph.

\section{REACTIONS OF HEPTA-ACETYL-4-GLUCOSIDO- $\gamma$ - METHYL-MANNOSIDE}

The ortho esters, such as trimethyl ortho acetic ester, are fairly resistant to alkaline hydrolysis, but they are very easily hydrolyzed by acids. Previously Fischer, Bergmann, and Rabe, as well as Dale, had found that one acetyl group in their gamma acetyl methyl glycosides was resistant to alkaline hydrolysis. Hepta-acetyl-4glucosido- $\gamma$-methyl-mannoside also exhibits this peculiar property which is characteristic of the ortho esters. Thus, six acetyl groups in the new substance are easily removed by either acids or alkalies, but the seventh is resistant to alkaline hydrolysis.

The reactions of these ortho sugar derivatives with hydrogen chloride are of unusual interest. Previously Dale had observed that a rapid change in specific rotation is produced by the addition of hydrogen chloride to a methyl alcoholic solution of tetra-acetyl- $\gamma$ methyl-mannoside. Under similar conditions the writer found an analogous change in the specific rotation of hepta-acetyl-4-glucosido$\gamma$-methyl-mannoside. Curves $I I I$ and $I I$ in Figure 1 illustrate the changes caused in the specific rotations of tetra-acetyl- $\gamma$-methylmannoside and hepta-acetyl-4-glucosido- $\gamma$-methyl-mannoside by the addition of two equivalents of hydrogen chloride in dry methyl alcoholic solution. In each case the rotation changes very quickly from a negative to a positive value. Previously it was believed that the change in rotation was caused by a shift in ring structure involving a migration of an acetyl group. ${ }^{12}$ The evidence for this interpretation was the experimental fact, discovered by Dale, ${ }^{13}$ that tetra-acetyl- $\beta$ methyl-mannoside may be obtained from tetra-acethyl- $\gamma$-methylmannoside after treatment with an alcoholic solution of hydrogen chloride. The hydrogen chloride was removed with silver carbonate and the resulting solution gave about a 7 per cent yield of crystalline tetra-acetyl- $\beta$-methyl-mannoside. By the same procedure the writer was able to obtain hepta-acetyl-4-glucosido- $\beta$-methyl-mannoside from the gamma acetyl methyl glycoside. The isolation of these products does not explain the change in rotation observed after the addition of hydrogen chloride because the specific rotations of the beta-acetyl methyl glycosides are more negative than the rotations of the corresponding gamma derivatives. Obviously, in order to change the rotation from a negative value to a positive value, a substance, or substances, of positive rotation must be formed. The reaction may involve many factors, including the solvent and silver carbonate; hence the experimental conditions were altered so as to eliminate the use of silver carbonate and methyl alcohol. When a chloroform solution of hepta-acetyl-4-glucosido- $\gamma$-methyl-mannoside is treated with hydrogen chloride, a rapid change in rotation occurs, as may

11 Bott, Haworth, Hirst, J. Chem. Soc., p. 1395; 1930.

13 Hudson, J. Am. Chem. Soc., vol. 48, p. 1433; 1926.

is See footnote 4, p. 1116 . 
be seen in Curve $I$ of Figure 1. This change in rotation is similar to that observed with alcoholic solutions. The chloroform solution gives on evaporation chloro-hepta-acetyl-4-glucosido-mannose. This substance gives a positive rotation which explains the changes in rotation previously noted. Inasmuch as the methyl glycosides are conveniently prepared by the use of a small quantity of hydrogen chloride in methyl alcoholic solution, one would not expect a true methyl glycoside to yield the chloro-acetyl derivative under the conditions given above.

Before turning to the interpretation of this reaction in relation to the ortho ester structure, we will consider the reaction of heptaacetyl-4-glucosido- $\gamma$-methyl-mannoside with moist hydrogen chloride. When hepta-acetyl-4-glucosido- $\gamma$-methyl-mannoside is treated, first with dry hydrogen chloride in methyl alcohol, and then with moist

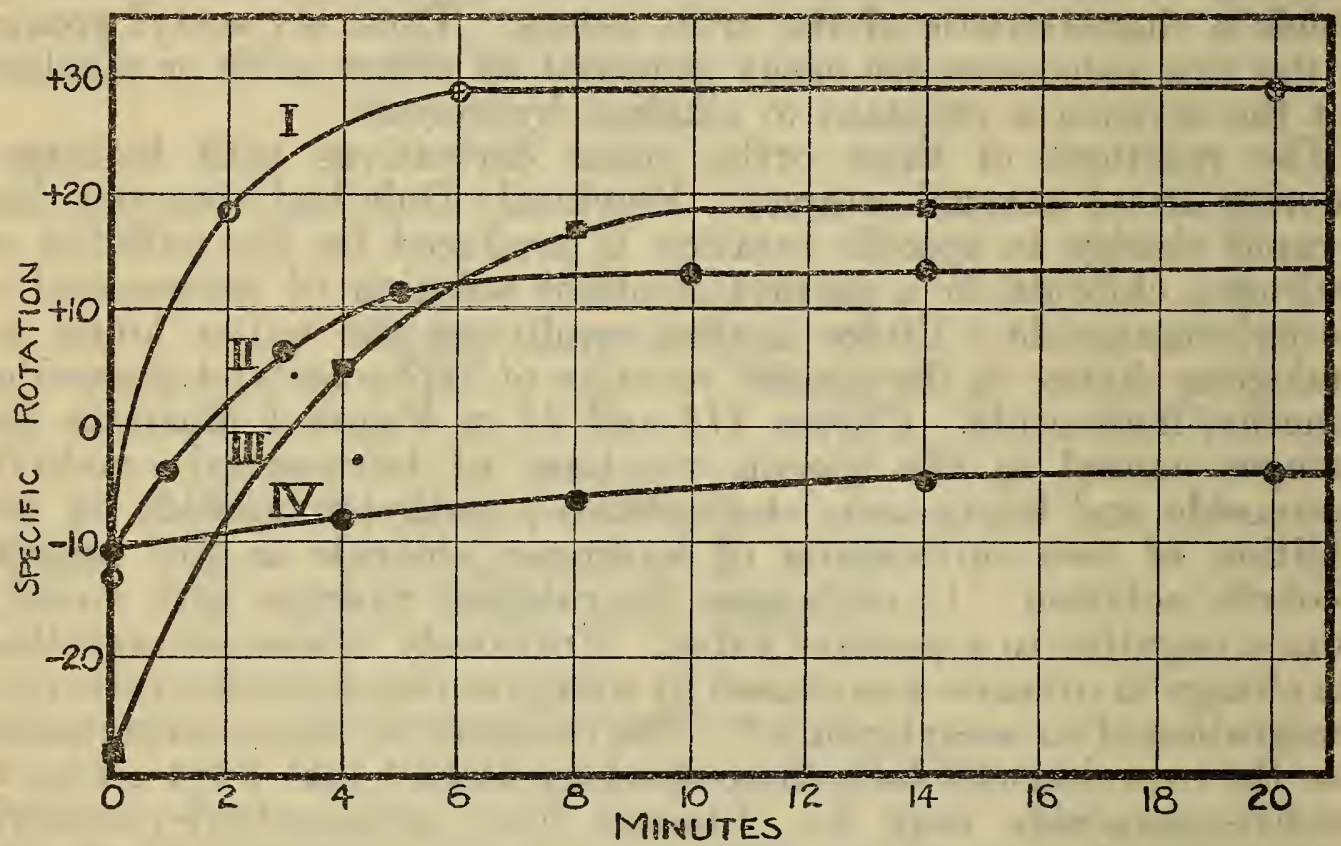

FIGURE 1.-Changes in specific rotation after the addition of iwo equivalents of hydrogen chloride to $0.05 \mathrm{~N}$ solutions of the acetylated $\gamma$-methyl glycosides

I, Hepta-acetyl-4-glucosido- $\gamma$-methyl-mannoside in chloroform.

II, Hepta-acetyl-4-glucosido- $\gamma$-methyl-mannoside in methyl alcohol.

III, Tetra-acetyl- $\gamma$-methyl-mannoside in methyl alcohol.

$I V$, Hepta-acetyl-4-glucosido- $\gamma$-methyl-mannoside in 80 per cent aqueous methyl alcohol.

silver carbonate, hexa-acetyl-4-glucosido-mannose ${ }^{14}$ is obtained in good yield.

The structure of the new hexa-acetyl-4-glucosido-mannose has not been rigidly determined. ${ }^{15}$ Since the normal acetylated methyl glycosides are not appreciably altered, under the conditions used in the preparation of the hexa-acetate, probably the six normal acetyl groups in the parent hepta-acetyl-4-glucosido- $\gamma$-methyl-mannoside would remain intact. Inasmuch as the ortho acetates are very easily decomposed by acids the simplest explanation for the formation of the hexa-acetyl-4-glucosido-mannose is that the ortho acetate group is

14 This product was obtained in an attempt to prepare ortho-hepta-acetyl-4-glucosido-mannose, and it was reported as such in a preliminary note in the J. Am. Chem. Soc., vol. 52, p. 5298; 1930.

16 What appears to be ananalogous derivative of mannose has been obtained by the method used in the preparation of hexa-acetyl-4-glucosido-mannose. It is anticipated that this substance may be used for the synthesis of disaccharides and other sugar derivatives. These phases of the subject as well as the structure of the substance are being investigated. 
split off. This would give a 1,2 unsubstituted hexa-acetyl-4-glucosido-mannose. This substance contains a free carbonyl group, and, hence, one would expect it to exhibit mutarotation. 'The new hexaacetate does not exhibit mutarotation in methyl alcohol or glacial acetic acid, but it does exhibit a very slight mutarotation in pyridine. The absence of mutarotation, in the solvents first mentioned, is exceptional if the substance is a single form of the surar containing a free reducing group. On acetylation with acetic anhydride and pyridine at low temperatures it gives a good yield of $\alpha$-octa-acetyl-4glucosido-mannose. Since these reagents do not usually cause any marked changes in structure, the formation of this normal octaacetate indicates that the original substance is a normal hexa-acetate instead of an or tho acetate. This conclusion is also supported by the fact that the hexa-acetate, as well as the octa-acetate, gives on hydrolysis with acid the same acetyl value as is obtained with alkali. Normal acetates give like values on hydrolysis with acids and alkalies, but usually ortho acetates give different values.

Hexa-acetyl-4-glucosido-mannose may be obtained also without the use of moist silver carbonate by the addition of water to the solution of hepta-acetyl-4-glucosido- $\gamma$-methyl-mannoside which has been previously treated with dry hydrogen chloride dissolved in methyl alcohol. But when hepta-acetyl-4-ghcosido- $\gamma$-methyl-mannoside was treated with an aqueous methyl alcoholic solution of hydrogen chloride no crystalline hexa-rcetate was obtained. 'The reaction was incomplete because a portion of the hepta-acetyl-4glucosido- $\gamma$-methyl-mannoside was reclaimed unchanged. The change in the optical rotation of the aqueous alcoholic solution is illustrated in Curve $I V$, Figure 1, page 111s. It is evident from the curve that the change is less rapid than that found for the chloroform and anhydrous methyl alcoholic solutions.

'The fact that hydrogen chloride reacts with hepta-acetyl-4-glucosido- $\gamma$-methyl-mannoside more rapidly and completely in anhydrous solvents, such as chloroform or dry methyl alcohol, than in alcoholic solutions containing water indicates that the reaction is not a simple hydrolysis depending upon the hydrogen-ion concentration. Probably the first step in the reaction is the formation of an addition product between the hydrogen chloride and one of the oxygen atoms. This addition product would account for the inhibiting action of water because the water would combine with the hydrogen chloride and thus reduce its tendency to combine with the gamma-acetylmethyl-glycoside. ${ }^{16}$

Since the hydrogen chloride might react with any one of the three oxygen atoms in the ortho ester group the reaction could proceed in several ways. If the hydrogen chloride united with the oxygen atom combined with the first carbon atom of the sugar, the addition product would give chloro-hepta-acetyl-4-glucosido-mannose by the elimination of methyl alcohol. This explains the formation of the chloro-acetyl derivative in chloroform solution. On the other hand, if the reaction be reversible, the addition of a considerable quantity of methyl alcohol would decrease the tendency to eliminate methyl alcohol, hence, under these conditions the chloro-acetyl derivative

10 The inhibiting effect of the water in this case is somewhat analogous to the inhibiting effect of water on the addition reactions of anhydrous auric chloride. (Kharasch and Isbell, J. Am. Chem. Soc., vol. 53, p. 3055 ; 1931.) 
would not be formed in as large amounts. When methyl alcohol was used as a solvent it was not possible to obtain any crystalline chlorohepta-acetyl-4-glucosido-mannose, but even so, a small quantity of the chloro-acetyl derivative might have been formed which would react with the dry silver carbonate to give the hepta-acetyl-4-glucosido- $\beta$-methyl-mannoside which was separated in small amounts. Furthermore, by reaction with water the hydrogen chloride addition product might be decomposed with the elimination of hydrogen chloride and methyl acetate to give hexa-acetyl-4-glucosido-mannose. The reactions are illustrated below.

Another reaction of hepta-acetyl-4-glucosido- $\gamma$-methyl-mannoside, somewhat analogous to that with hydrogen chloride, was observed in

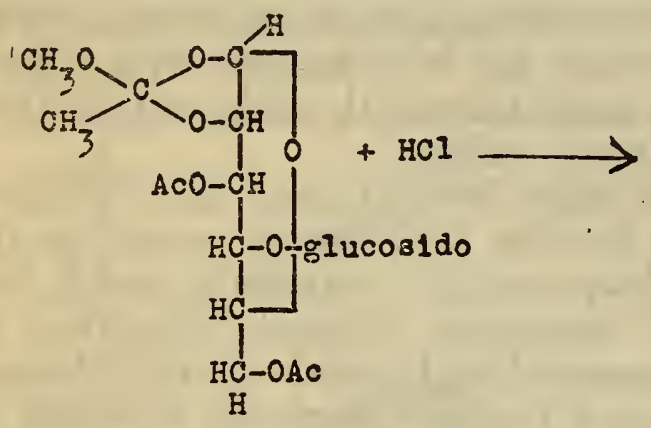

Hepta-acety 1-4-glucos1do

$y$ methyl-mannoside

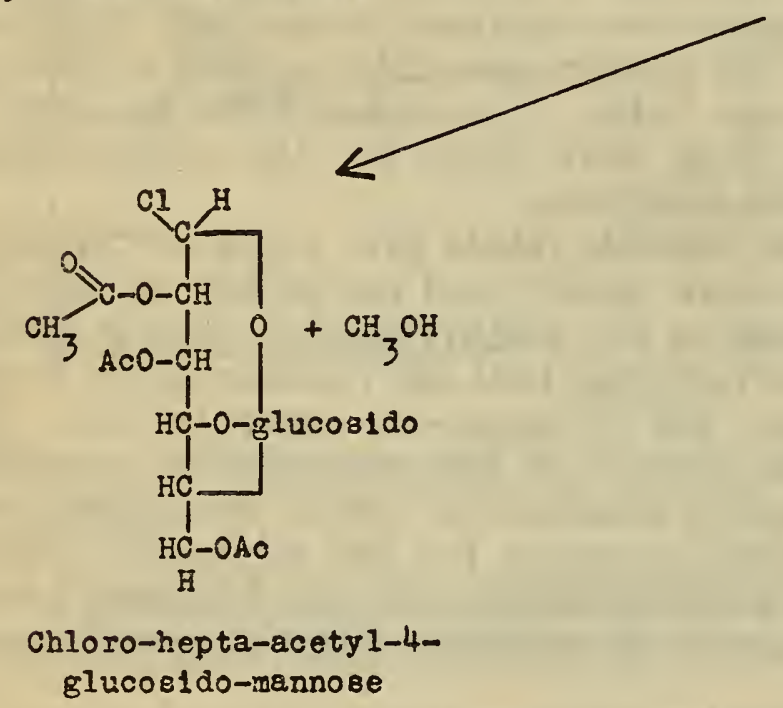

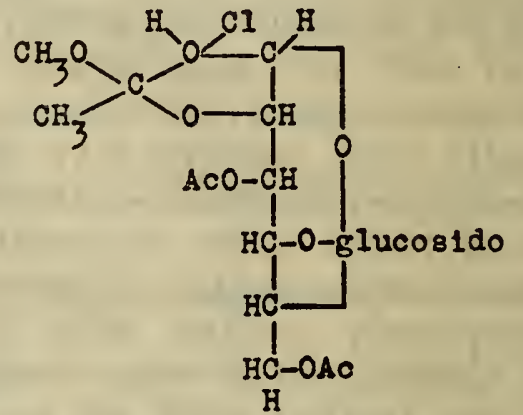

Hypothetical addition

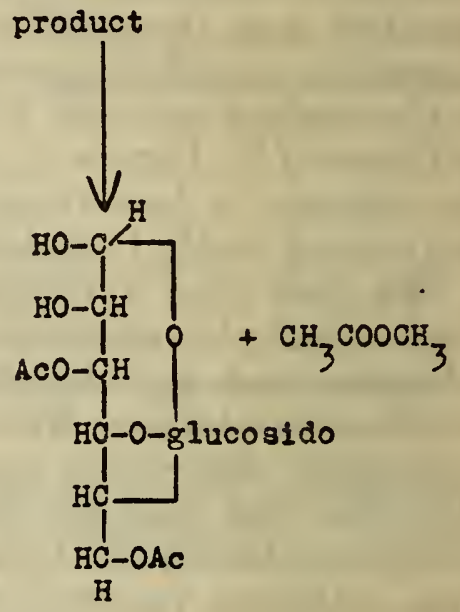

Hexa-acetyl-4-glucos1domannose

an attempt to prepare the acetylated alpha methyl glycoside. A chloroform solution of hepta-acetyl-4-glucosido- $\gamma$-methyl-mannoside was treated with titanium tetrachloride in the manner which Pacsu ${ }^{17}$ has found gives a satisfactory transformation of hepta-acetyl- $\beta$ methyl-cellobioside in to the corresponding alpha isomer. The product was not the isomeric alpha derivative, but chloro-hepta-acetyl4-glucosido-mannose. ${ }^{18}$ Thus it is seen that the gamma-acetylmethyl glycosides on treatment with titanium tetrachloride do not give the reactions characteristic of the true acetyl methyl glycosides.

17 Pascu, J. Am. Chem. Soc., vol. 52, p. 2571; 1930.

18 This reaction is somewhat similar to the reaction of titanium tetrachloride with the fully acetylated sugar which has been studied by Pacsu (Ber., vol. 61, p. 1508; 1928). 


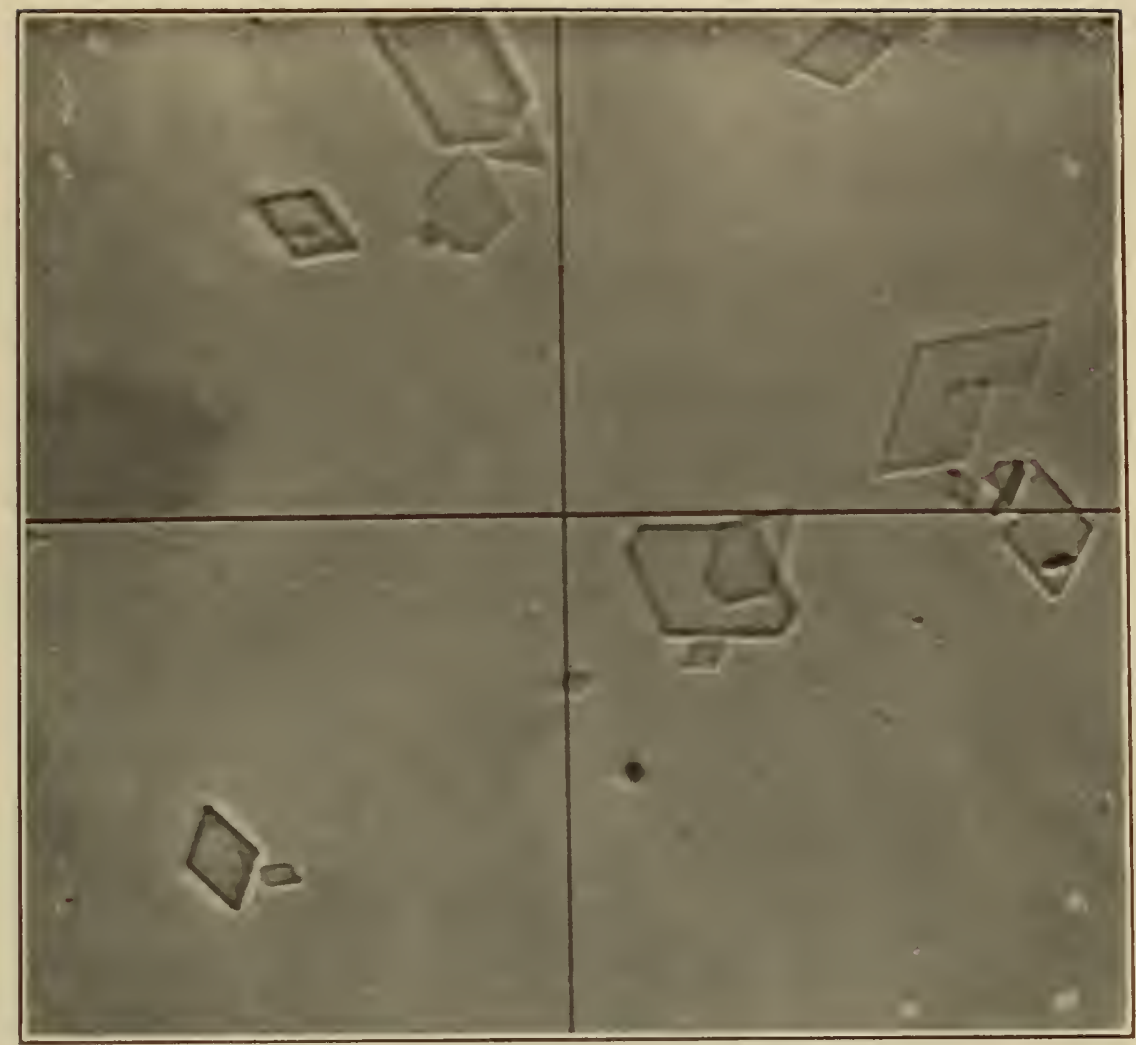

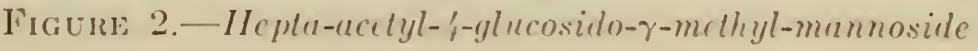

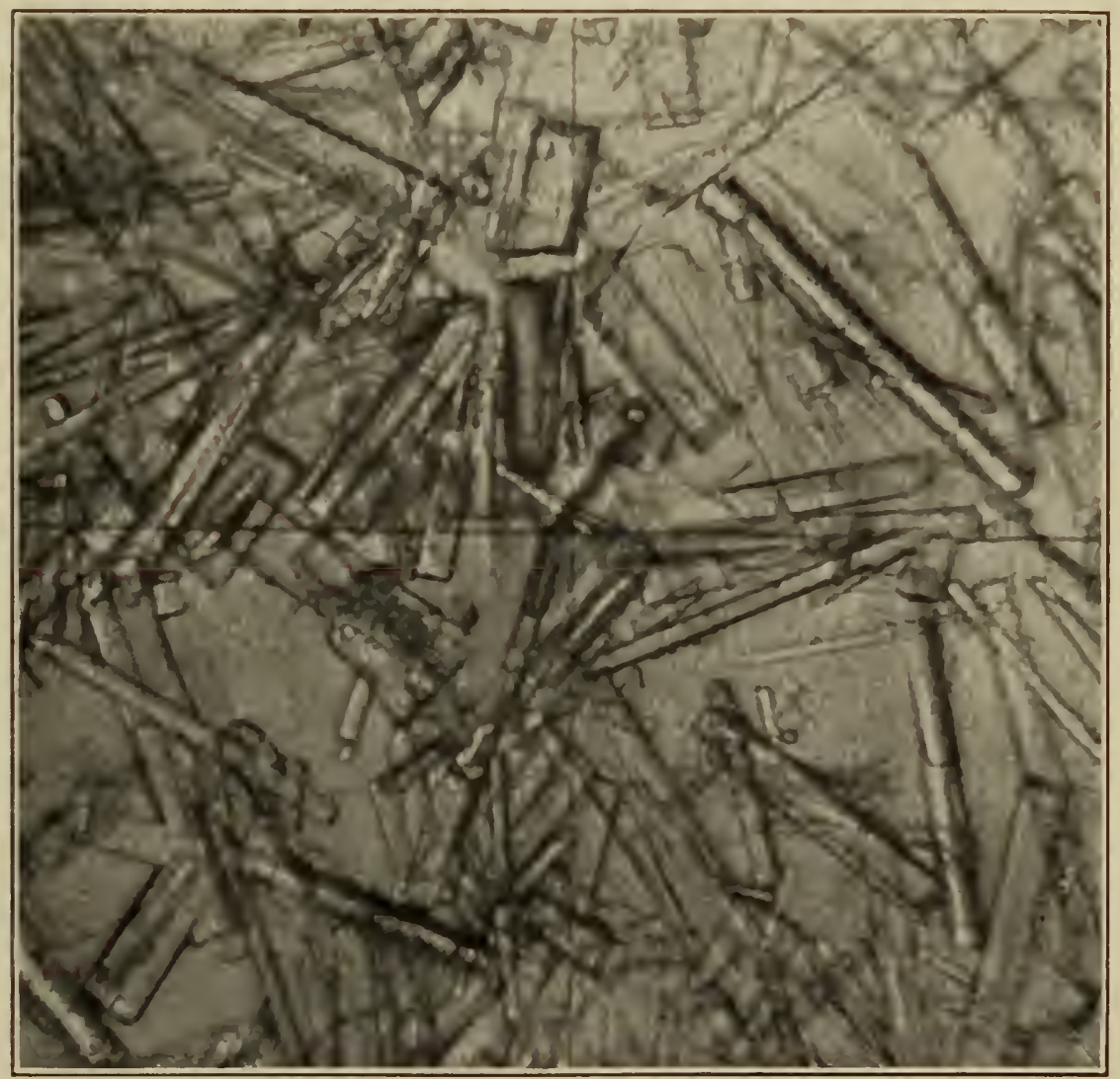

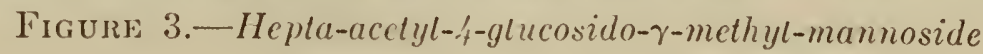
Crystallized from methyl alcohol. 
Another peculiar property of hepta-acetyl-4-glucosido- $\gamma$-methyl-. mannoside is that it gives two decidedly different crystalline forms. It was first obtained in thin tabular crystals, illustrated in Figure 2. When these crystals are dissolved in the proper quantity of methyl alcohol and allowed to stand a few minutes, a second crystalline form separates from the solution. The second form crystallizes in slender rectangular prisms, as shown in Figure 3. The latter substance may be reconverted into the first by recrystallization from hot amyl alcohol. After mild drying both substances give essentially the same melting points and specific rotations. The existence of this change in form, whatever it may be, suggests that the sugar derivatives exist in solution in various forms, perhaps, loosely combined with the solvent and that the structure of the substance which crystallizes from solution may be determined at least occasionally by the solvent employed. ${ }^{19}$

\section{CORRELATION OF THE DERIVATIVES OF 4-GLUCOSIDO- MANNOSE WITH THE DERIVATIVES OF MANNOSE}

The optical rotations of many of the derivatives of mannose do not follow the same numerical relationships which are found in the glucose series. ${ }^{20}$ The exceptional rotations show that either there are unusual differences in structure or else the theory of optical superposition is not valid. In a previous publication ${ }^{21}$ it was shown that the optical rotations of 4 -glucosido- $\alpha$-mannose and 4 -glucosido- $\beta$ mannose parallel the rotations of $\alpha$-and- $\beta$-mannose, which indicates that 4-glucosido- $\alpha$-mannose has the $\alpha$-mannose structure while 4 -glucosido- $\beta$-mannose has the $\beta$-mannose structure. In this paper additional derivatives of 4 -glucosido-mannose are reported and correlated with the derivatives of mannose. The existence of these derivatives of 4-glucosido-mannose, a sugar which can not form a 1,4 ring, clearly shows that the exceptional rotations are not caused by the 1, 4 ring structure.

TABLE 1.-Acetyl methyl glycosides of mannose, rhamnose, and 4-glucosido-mannose

\begin{tabular}{|c|c|c|c|c|c|}
\hline 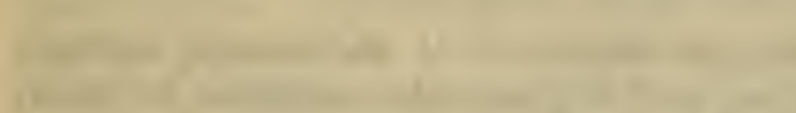 & Type & $\begin{array}{c}\text { Melt- } \\
\text { ing } \\
\text { point }\end{array}$ & $\begin{array}{l}\text { Molec- } \\
\text { ular } \\
\text { weight }\end{array}$ & {$[\alpha]_{D}^{a}$} & [M] \\
\hline 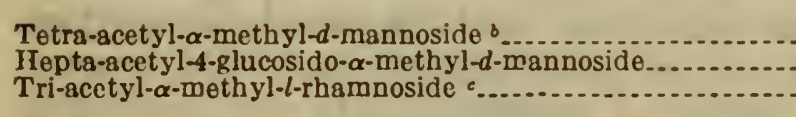 & $\begin{array}{l}1, \mathrm{~A} \\
1, \mathrm{~A} \\
1, \mathrm{~A}\end{array}$ & $\begin{array}{r}65 \\
185 \\
87\end{array}$ & $\begin{array}{l}362 \\
650 \\
304\end{array}$ & $\begin{array}{l}+49.1 \\
+29.3 \\
-53.5\end{array}$ & $\begin{array}{r}+17,770 \\
+19,050 \\
+-16,260\end{array}$ \\
\hline 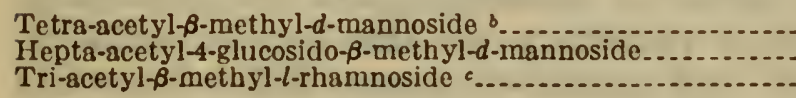 & $\begin{array}{l}1, \mathrm{~B} \\
\mathrm{~B}, \mathrm{~B} \\
\mathrm{1}, \mathrm{B}\end{array}$ & $\begin{array}{l}161 \\
178 \\
152\end{array}$ & $\begin{array}{l}362 \\
650 \\
304\end{array}$ & $\begin{array}{l}-46.8 \\
-23.2 \\
+45.7\end{array}$ & $\begin{array}{r}-16,940 \\
-15,080 \\
d+13,890\end{array}$ \\
\hline 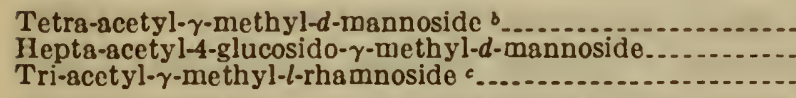 & $\begin{array}{l}1, \mathrm{C} \\
1, \stackrel{\mathrm{C}}{\mathrm{C}}\end{array}$ & $\begin{array}{r}105 \\
167 \\
85\end{array}$ & $\begin{array}{l}362 \\
650 \\
304\end{array}$ & $\begin{array}{l}-26.6 \\
-12.7 \\
+28.0\end{array}$ & $\begin{array}{r}-9,630 \\
-8,260 \\
8+8,510\end{array}$ \\
\hline
\end{tabular}

- The values given are for chloroform solutions except for the derivatives of rhamnose which are given for acetylene tetra-chloride solutions.

$\checkmark$ Dale, J. Am. Chem. Soc., vol. 46, p. 1046; 1924.

- Fischer, Bergmann, and Rabe, Ber., vol. 53, p. 2362; 1920.

$\checkmark$ Reversing the signs of the rotations of the $l$-rhamnose derivatives gives the rotations in the $d$-rhamnose series. Such rotations are comparable with the rotations of the $d$-mannose, and 4-glucosido- $d$-mannose derivatives, because $d$-rhamnose and $d$-mannose have similar structures.

19 In a previous publication (B. S. Jour. Research, vol. 5 (RP226), p. 748) the writer has shown that the equilibrium between the various forms of $d$-gulose $\mathrm{CaCl}_{2}$ may be altered by changing the solvent.

${ }_{20}$ Hudson, J. Am. Chem. Soc., vol. 48, p. 1434; 1926.

» See footnote 1, p. 1115. 
TABLE 2.-Acetyl derivatives of mannose and 4-glucosido-mannose

Fluoro-tetra-acetyl-d-mannose 1

Fluoro-hepta-acetyl-4-glucosido-mannose

Chloro-tetra-acetyl- $d$-mannose 3

Chloro-bepta-acetyl-4-glucosido-d-mannose ${ }^{2}$

Bromo-tetra-acetyl-d-mannose 1

Bromo-hepta-acetyl-4-glucosido-d-mannose ${ }^{2}$

Iodo-tetra-acetyl-d-mannose 1

Iodo-hepta-acetyl-4-glucosido-d-mannose 2

$\alpha$-Penta-acetyl-d-mannose 4

$\alpha$-Octa-acetyl-4-glucosido- $d$-mannose 2

$\beta \cdot P e n t a-a c e t y l-d$-mannose 4

$\beta$-Octa-acetyl-4-glucosido-d-maniose

\begin{tabular}{|r|r|r}
$\begin{array}{c}\text { Molec- } \\
\text { ular } \\
\text { weight }\end{array}$ & {$[\alpha]_{\mathrm{D}}$} & \multicolumn{1}{c}{$[\mathrm{M}]$} \\
\hline 350 & +21.5 & $+7,530$ \\
\hline 638 & +13.6 & $+8,680$ \\
367 & +89.5 & $+32,850$ \\
655 & +51.2 & $+33,540$ \\
411 & +131.6 & $+54,090$ \\
699 & +77.9 & $+54,450$ \\
458 & +190.5 & $+87,250$ \\
746 & +111.5 & $+83,180$ \\
390 & +55 & $+21,450$ \\
678 & +36.2 & $+24,540$ \\
390 & -25.3 & $-9,870$ \\
678 & -13.0 & $-8,810$ \\
& &
\end{tabular}

1 Brauns, B. S. Jour. Research, vol. 7 (RP358), p. 573; 1931.

\& Brauns, J. A m. Chem. Soc., vol. 48, p. 2776; 1926.

3 Brauns, J. A m. Chem. Soc., vol. 44, p. 401; 1922.

4 Hudson and Dale, J. Am. Chem. Soc., vol. 37, p. 1280; 1915.

TABLE 3.-Molecular rotations of mannose derivatives
$\alpha-d-M a n n o s e !$

4-Glucosido- $\alpha$-mannose 2 (monohydrate)

6- $d$-Mannose ${ }^{3}$

4-Glucosido- $\beta$-mannose.

$\alpha$-Methyl-d-mannoside

4-Glucosido- $\alpha$-methyl mannoside

\begin{tabular}{|r|c|c}
$\begin{array}{c}\text { Molec- } \\
\text { ular } \\
\text { weight }\end{array}$ & {$[\alpha]_{\mathrm{D}}$} & \multicolumn{1}{c}{$[\mathrm{M}]$} \\
\hline 180 & +30 & $+5,400$ \\
360 & +14.6 & $+5,260$ \\
180 & -17 & $-3,060$ \\
342 & -6.5 & $-2,220$ \\
194 & +79 & $+15,330$ \\
356 & +46 & $+16,380$ \\
\end{tabular}

1 Levene, J. Biol. Chem., vol. 57, p. 329; 1923; vol. 59, p. 129; 1924.

I Isbell, B. S. Jour. Research, vol. 5 (RP253), p. 1186; 1930.

3 Hudson and Yanovski, J. Am. Chem. Soc., vol. 39, p. 1013; 1917.

4 Van Ekenstein, Rec. trav. chim., vol. 15, p. 223; 1896.

5 Haworth, Hirst, Streight, Thomas, and Webb, J. Chem. Soc., p. 2639; 1930.

A comparison of the molecular rotations of the acetyl methyl glycosides of mannose, rhamnose, and 4-glucosido-mannose is given in Table 1. Each of the three sugars forms three acetyl methyl glycosides exhibiting analogous chemical and physical properties. It is apparent, without any involved calculations, that the derivatives of the various sugars bear the same relationship to one another in the three series. The molecular rotations of the $\alpha-, \beta$, and $\gamma-$ acetylated methyl glycosides of 4-glucosido-mannose differ from the molecular rotations of the $\alpha, \beta$, and $\gamma$ mannose derivatives by $+1,280$, $+1,860$, and $+1,370$, respectively. These values show that the acetylated 4-glucosido-mannose chain ${ }^{22}$ is slightly more dextrorotatory than the mannose chain.

As illustrated in Table 2 this correlation in molecular rotations is not restricted to the acetylated methyl glycosides, but is also found in the halogen acetyl derivatives and in the acetylated sugars. The molecular rotations of the halogen acetyl derivatives of 4 -glucosido-

23 The rotation of the 4-glucosido-inannose chain is composed of the rotation of the primary mannose group and the secondary glucose residue. The approximate agreement of its molecular rotation with the rotation of the mannose chain is the result of compensation; that is, tho dextro rotating glucose residue is compensated for by its $\beta$.glucosidic union. 
mannose differ from the molecular rotations of the corresponding derivatives of mannose by $+1,150,+690,+360$, and $-4,070$, respectively, for the fluorides, chlorides, bromides, and iodides. These values are of the same order as those obtained from the acetylated glycosides and indicate that the rotation of the acetylated 4glucosido-mannose chain is only slightly different from the rotation of the acetylated mannose chain. However, the difierences between the rotations of corresponding derivatives of 4-glucosido-mannose and mannose decrease progressively from fluorine to iodine. This is additional evidence in support of the writer's previous statement ${ }^{23}$ that a change in the first carbon produces small but definite changes in the rotation of the rest of the molecule. The molecular rotation of penta-rcetyl-mannose $(+21,450)$ has been considered exceptional, ${ }^{24}$ hence the comparison of its rotation with the rotation of the closely related substance, octa-rcetyl-4-glucosido-mannose $(+24,540)$, is of particular interest. The difference in the molecular rotations of the substances just mentioned $(+3,090)$, as well as the difference between the molecular rotations of the $\beta$-forms $(+1,060)$, is in fair agreement with the values derived from the acetylated methyl glycosides and from the halogen acetyl derivatives.

The rotations of some unacetylated derivatives of mannose and 4glucosido-mannose are given in Table 3 . The differences between the molecular rotations of the corresponding derivatives of 4-glucosidomannose and mannose are $-140,+840$, and $+1,050$, respectively, for the $\alpha$-and $\beta$-forms of the sugars and the $\alpha$-methyl glycosides. The average of these differences shows that the unacetylated derivatives of 4-glucosido-mannose are usually slightly more dextrorotatory than the corresponding derivatives of mannose. It will be recalled that a similar relation was found for the acetyl derivatives.

The difference between the molecular rotations of the $\alpha$ - and $\beta$-forms of 4 -glucosido-mannose is $+7,480$. This value is of the same order as the value $+8,460$ obtained for the difference in the molecular rotations of $\alpha$ - and $\beta$-mannose. The latter value has long been recognized as different from the value $+17,000^{25}$ obtained from many other sugar derivatives. Anomalous values are also found for the acetylated methyl glycosides. The differences between the molecular rotations (Table 1) of the $\alpha$-and $\beta$-acetyl methyl glycosides of mannose, rhamnose, and 4 -glucosido-mannose are $+34,710,+30,150$, and $+34,130$, respectively, which are widely different from the values $+53,900,+53,460$, and $+52,300$, obtained from the $\alpha$-and $\beta$-acetyl methyl glucosides, galactosides, and xylosides. ${ }^{26}$ Similar variations are found in the differences between the molecular rotations of the acetylated sugars. Thus the difference between the molecular rotations of the two forms of octa-acetyl-4-glucosido-mannose is $+33,350$, which is of the same order as the value $(+31,200)$ obtained by Hudson ${ }^{27}$ from the corresponding derivatives of mannose. These values are also close to the value $(+32,700)$ derived by him from one pair of his $d$-galactose penta-acetates, ${ }^{28}$ but they differ widely from the values obtained from many other sugars.

\footnotetext{
23 Isbell, B. S. Jour. Research, vol. 5 (RP253), p. 1184; 1930.

34 Hudson, J. Am. Chem. Soc., vol. 48, p. 1433; 1926.

28 Hudson, J. A m. Chem. Soc., vol. 48, p. 1424; 1926.

20 Dale and Hudson, J. Am. Chem. Soc., rol. 52, p. 2534; 1930

27 Hudson, B. S. Sci. Paper No. 533, p. 259; 1926 . viously beljeved, but that they difier in some manner as yet not clearly understood.
} 
The difference between the molecular rotations of 4 -glucosido- $\alpha$ methyl-mannoside $(+16,380)$ and 4 -glucosido- $\beta$-methyl-mannoside $(-17,700)$ is approximately $+34,000$. This value is only slightly less than the value $(+37,500)$ obtained in the glucose series. Since $\beta$-methyl-mannoside is not known in the crystalline state the analogous value in the mannose series is not available. Inasmuch as the 4 -glucosido- $\beta$-methyl-mannoside is somewhat peculiar, in that it seemingly contains 1 molecule of water for each 2 molecules of the glycoside, further experimental work is necessary before any conclusions may be drawn from its rotation.

In the preceding pages it has been shown that the rotations of certain derivatives of 4-glucosido-mannose, corresponding to the derivatives of mannose which give abnormal rotations, are likewise unusual in respect to the numerical relations which are obtained from many other sugars. The existence of the derivatives of 4glucosido-mannose which give abnormal rotations clearly shows that these "exceptional" values are not caused by a 1, 4 oxygen ring.

\section{EXPERIMENTAL DETAILS}

\section{HEPTA-ACETYL-4-GLUCOSIDO- $\gamma$-METHYL-MANNOSIDE}

Twenty-five grams of bromo-hepta-acetyl-4-glucosido-mannose was mixed at $0^{\circ} \mathrm{C}$. with $25 \mathrm{~g}$ of dry freshly prepared silver carbonate in $250 \mathrm{ml}$ of anhydrous methyl alcohol. After being shaken for 30 minutes the solution was filtered. The filtrate, which gave a negative halogen test, was concentrated in vacuo to a thick sirup. After the addition of a small quantity of ether a crystalline product was separated. As shown in Figure 2, the substance crystallizes under these conditions in plates which frequently appear as diamonds or hexagons. This substance is hepta-acetyl-4-glucosido- $\gamma$-methyl-mannoside. The mother liquor was evaporated to a sirup from which several small fractions of crystalline material were obtained by alternate addition of ethyl alcohol and evaporation. Seventeen grams of crystalline material was obtained, which contained the $\beta$ and $\gamma$ isomers. By fractional crystallization from ethyl alcohol $14 \mathrm{~g}$ of the $\gamma$ compound was separated. After drying at $50^{\circ}$ in vacuo it melted at $167^{\circ} \mathrm{C}$. and gave a specific rotation of -12.9 . After two additional crystallizations it melted at the same temperature and in chloroform solution gave $[\alpha]_{D}^{20}=-12.7(0.6432 \mathrm{~g}$ substance dissolved in $20 \mathrm{ml}$ of chloroform read $-2.36^{\circ} \mathrm{S} ., 2-\mathrm{dm}$ tube). Analysis calculated for $\mathrm{C}_{27} \mathrm{H}_{38} \mathrm{O}_{18}$ : $\mathrm{C}, 49.82 ; \mathrm{H}, 5.89$. Found: $\mathrm{C}, 50.07 ; \mathrm{H}, 5.96$.

The compound $(0.4046 \mathrm{~g})$ was refluxed 4 hours with $50 \mathrm{ml}$ of 0.25 $N$, sulphuric acid. The back titration with standard alkali gave a value which was equivalent to an increase of $43.3 \mathrm{ml}$ of $0.1 \mathrm{~N}$ acid. This equals 64.25 per cent acetic acid, while that calculated for completely hydrolyzed hepta-acetyl-4-glucosido-methyl-mannoside is 64.62 per cent. Hydrolysis with alkalies gave a different result. The percentage of acetic acid obtained by alkaline hydrolysis corresponds to the quantity calculated by assuming the hydrolysis of only six acetyl groups. A $0.1417 \mathrm{~g}$ sample was shaken with $50 \mathrm{ml}$ of $0.1 \mathrm{~N}$ barium hydroxide solution for 2 hours and allowed to stand for 18 hours in the refrigerator. The back titration with standard hydrochloric acid showed that $13.1 \mathrm{ml}$ of $0.1 \mathrm{~N}$ acid was formed by 
hydrolysis. This equals 55.50 per cent acetic acid while that calculated for the hydrolysis of six acetyl groups is 55.39 per cent.

Hepta-acetyl-4-glucosido- $\gamma$-methyl-mannoside $(0.4137 \mathrm{~g})$ was dissolved in sufficient methyl alcohol to give a volume of $11 \mathrm{ml}$; the specific rotation was then -10.5. After the addition of $0.5 \mathrm{ml}$ of methyl alcohol, containing $0.043 \mathrm{~g}$ of hydrogen chloride, the following specific rotations were observed after $1,3,5$, and 10 minutes, respectively: $-4.1,+7.2,+11.2$, and +12.8 . After 10 minutes the rotation was virtually constant as no further change was observed during the day.

The tabular crystals of hepta-acetyl-4-glucosido- $\gamma$-methyl-mannoside on recrystallization from methyl alcohol give a second crystalline product which separates in slender rectangular prisms. About $0.35 \mathrm{~g}$ of finely powdered hepta-acetyl-4-glucosido- $\gamma$-methyl-mannoside was dissolved in $5 \mathrm{ml}$ of methyl alcohol. Solution was complete in about one minute and in a few minutes a new crystalline folm of heptaacetyl-4-glucosido- $\gamma$-methyl-mannoside separated from the solution. (Fig. 3.) It was collected upon a filter and washed with a small quantity of methyl alcohol. On evaporation of the mother liquor additional material was obtained, the final yield being nearly quantitative. The substance after drying one hour at $40^{\circ}$ in vacuo melted at $165^{\circ}$ to $167^{\circ}$ (not sharp), and in chloroform $[\alpha]_{D}^{20}=-13.2$ (0.3408 g substance dissolved in $10 \mathrm{ml} \mathrm{read}-2.60^{\circ} \mathrm{S}$. in a $2 \mathrm{dm}$ tube). Analysis calculated for $\mathrm{C}_{27} \mathrm{H}_{35} \mathrm{O}_{18}$ : $\mathrm{C}, 49.82$, $\mathrm{H} ; 5.89$. Found: $\mathrm{C}$, $49.73 ; \mathrm{H}, 5.98$.

A quantity of the prismatic crystals was dissolred in a sinall amount of hot amyl alcohol; on cooling the substance separated in tabular crystals which seemed identical with the form first described. The tabular form was also obtained by recrystallizing the prismatic form from chloroform and from ether.

\section{HEPTA-ACETYL-4-GLUCOSIDO- $\beta$-METHYL-MANNOSIDE}

The material which remained after the crystallization of the $\gamma$ compound consisted of the $\beta$ derivative nixed with some $\gamma$ compound. The $\beta$ compound is slightly less soluble in water and in ethyl alcohol than the $\gamma$ compound, but even so the separation of the two substances is difficult. The nearly pure $\beta$ compound was obtained by crystallizing the impure product, first from water and then from absolute ethyl alcohol. It crystallizes in prisms which are frequently very slender, but under farorable conditions they become wider and thicker. The melting point is $178^{\circ}$ and the taste very bitter.

The product, after drying at $50^{\circ}$ in vacuo, gave in chloroform solution $[\alpha]_{D}^{2 n}=-22.3(0.6563 \mathrm{~g}$ of the compound dissolved in $25 \mathrm{ml}$ read $-3.38^{\circ} \mathrm{S}$. in a $2 \mathrm{dm}$ tube). Analysis calculated for $\mathrm{C}_{27} \mathrm{H}_{38} \mathrm{O}_{18}$ : C, 49.82; H, 5.89. Found: C, 49.54; H, 5.75.

As described in the next paragraph hepta-acetyl-4-glucosido- $\beta$ methyl-mannoside was deacetylated and crystalline 4-glucosido- $\beta$ methyl mannoside was prepared. This substance on acetylation regenerates pure hepta-acetyl-4-glucosido- $\beta$-methyl-mannoside, which after recrystallization melts at $178^{\circ}$ and gives $[\alpha]_{D}^{20}=-23.2$. 


\section{4-GLUCOSIDO- $\beta$-METHYL-MANNOSIDE}

This substance was obtained from its hepta-acetate by deacetylation with barium methylate. ${ }^{29}$ The acetylated glycoside $(1.5 \mathrm{~g})$ was dissolved in $30 \mathrm{ml}$ of cold methyl alcohol; after the addition of $2 \mathrm{ml}$ of $0.4 \mathrm{~N}$ barium methylate it was allowed to stand 24 hours in the refrigerator. The barium was then precipitated with sulphuric acid and removed by filtration. The filtrate was evaporated in vacuo to a thick sirup from which the new substance separated in slender prismatic crystals. The crystalline material $(0.7 \mathrm{~g})$ was triturated with absolute ethyl alcohol and collected upon a filter. It was then recrystallized from 95 per cent alcohol. It is very soluble in water and moderately soluble in hot ethyl alcohol. After two crystallizations it melted at $229^{\circ} \mathrm{C}$. This meiting point was not changed by further crystallization. It gave in water solution $[\alpha]_{D}^{20}=-48.5$ $\left(0.4063 \mathrm{~g}\right.$ dissolved in $10 \mathrm{ml}$ read $-11.38^{\circ} \mathrm{S}$. in a $2 \mathrm{dm}$ tube. $)$ The specific rotation in water for the mercury green line $(\lambda=5,461)$ was found to be -55.7 .

The substance was dried at $60^{\circ}$ in vacuo. Analysis calculated for $\left(\mathrm{C}_{13} \mathrm{H}_{24} \cdot \mathrm{O}_{11}\right)_{2} \cdot \mathrm{H}_{2} \mathrm{O}: \mathrm{C}, 42.72 ; \mathrm{H}, 6.90$. Found: $\mathrm{C}, 42.90 ; 42.94 ; \mathrm{H}$, $6.83,6.83$. The analysis of the compound indicates that it is slightly impure or else that it contains 1 molecule of water for each 2 molecules of the glycoside. It does not lose water at $100^{\circ}$ in vacuo which indicates that the water, if present, is held tightly.

The new compound gives hepta-acetyl-4-glucosido- $\beta$-methyl-mannoside in good yield on acetylation. About $0.3 \mathrm{~g}$ of the substance was mixed with a cold solution of $2 \mathrm{ml}$ of acetic anhydride and 2.5 $\mathrm{ml}$ of pyridine and allowed to stand 20 hours in the refrigerator. The mixture was then poured into ice water, and the product separated by extraction with chloroform; after the customary washing, drying, and evaporation about $0.5 \mathrm{~g}$ of crystalline hepta-acetyl-4glucosido- $\beta$-methyl-mannoside was obtained. After recrystallization from ethyl alcohol it melted at $178^{\circ}$ and gave $[\alpha]_{D}^{20}=-23.2(0.4702$ $\mathrm{g}$ dissolved in $10 \mathrm{ml}$ of chloroform read-6.31 ${ }^{\circ} \mathrm{S}$. in a $2 \mathrm{dm}$ tube). This specific rotation is slightly more negative than that obtained for the sample of hepta-acetyl-4-glucosido- $\beta$-methyl-mannoside described in the previous paragraph. Inasmuch as the melting point of either sample is not lowered by the addition of the other the two products are the same; consequently the original hepta-acetyl-4-glucosido- $\beta$ methyl-mannoside was not fundamentally altered by deacetylation.

The deacetylated product is probably a hydrated 4-glucosido- $\beta$ methyl-mannoside. It will be investigated further, the small amount of material on hand preventing additional study at this time.

\section{HEPTA-ACETYL-4-GLUCOSIDO- $\alpha$-METHYL-MANNOSIDE}

This product was obtained from the mother liquor which remained after the separation of the $\beta$ and $\gamma$ isomers. After all of the solvent was removed the residue was triturated with about $25 \mathrm{ml}$ of absolute methyl alcohol and the mixture, which contained some crystalline material, was allowed to stand. After several days $0.5 \mathrm{~g}$ of crystalline material had separated. Under the microscope the crystals appear as thin diamond-shaped prisms. Analysis calculated for $\mathrm{C}_{27} \mathrm{H}_{38} \mathrm{O}_{18}$ : $\mathrm{C}, 49.82 ; \mathrm{H}, 5.89$. Found: $\mathrm{C}, 49.81 ; \mathrm{H}, 5.96$. After the product was 
recrystallized from methyl alcohol it melted at $185^{\circ}$ and gave $[\alpha]_{D}^{20}=$ +26.7. Two other preparations of the same substance melted at $184^{\circ}$ to $185^{\circ}$ and gave slightly higher specific rotations $(+28.9$, +28.5 ). They were combined and recrystallized from methyl alcohol. the resulting product melted at $185^{\circ}$ and gave $[\alpha]_{D}^{20}=+29.3$ in chloroform. $(0.2105 \mathrm{~g}$ of the compound dissolved in $10 \mathrm{ml}$ of chloroform read $+3.56^{\circ} \mathrm{S}$. in a $2 \mathrm{dm}$ tube.) The small amount of material on hand prevented further study of the substance which has also been prepared very recently by Haworth, Hirst, Streight, Thomas, and Webb. They report the melting point as $184^{\circ}$ and $[\alpha]_{D}^{20}=+30$.

A considerable amount of material remained after the separation of the $\alpha, \beta$, and $\gamma$ isomers. The methyl alcohol was remored and the residue (about $5 \mathrm{~g}$ ) was dissolved in amyl alcohol. The solution, after cooling to $0^{\circ}$, gave a low melting crystalline product. The crude product melted near room temperature and in chloroform solution gave $[\alpha]_{D}^{20}=+15$. This residual product may contain one or more additional substances which have not been separated.

\section{HEXA-ACETYL-4-GLUCOSIDO-MANNOSE}

A $5 \mathrm{~g}$ sample of hepta-acetyl-4-glucosido- $\gamma$-methyl-mannoside was suspended in $50 \mathrm{ml}$ of cold absolute methyl alcohol and $4 \mathrm{ml}$ of methyl alcohol containing $0.34 \mathrm{~g}$ of dry hydrogen chloride were added. The flask was shaken until solution was complete (about one minute) and then allowed to stand at room temperature. After 10 minutes the acid solution was mixed with a paste consisting of $10 \mathrm{~g}$ of silver carbonate and $2 \mathrm{ml}$ of water. The mixture was stirred until a negative test for halogens was obtained, when the silver salts were removed by filtration. The solution was next evaporated in vacuo to a thick sirup which crystallized in the course of several days. The new substance crystallizes in rectangular prisms. It is difficultly soluble in water, fairly soluble in ether, and very soluble in chloroform. The crude material $(3.8 \mathrm{~g})$ was recrystallized from hot othyl alcohol. The resulting product melted at $171^{\circ}$. 'The melting point is not very sharp, but it could not be changed by recrystallization from alcohol or from ethyl acetate by the addition of petroleum ether.

The optical rotation was determined in chloroform, methyl alcohol, glacial acetic acid and pyridine solutions. The recrystallized compound gave in chloroform solution $[\alpha]_{D}^{20}=+21.7(0.6040 \mathrm{~g}$ of the substance dissolved in $25 \mathrm{ml}$ read $+3.03^{\circ} \mathrm{S}$. in a 2 din tube); methyl alcohol, $[\alpha]_{D}^{20}=+14.6(0.5325 \mathrm{~g}$ of the substance dissolved in $25 \mathrm{ml} 4$ minutes after solution, read $1.80^{\circ} \mathrm{S}$. in a $2 \mathrm{dm}$ tube); in glacial acetic acid $[\alpha]_{D}^{20}=+18.5(0.3055 \mathrm{~g}$ of the substance dissolved in $10 \mathrm{ml}$, six minutes after solution, read $+3.31^{\circ} \mathrm{S}$. in a $2 \mathrm{dm}$ tube). The optical rotations of the solutions just given were read at intervals during the ensuing 24 hours and no changes were detected. However, in pyridine solution a sinall change was observed. The sample $(0.2338 \mathrm{~g})$ was dissolved in sufficient pyridine to give $10 \mathrm{ml}$ of solution. The following specific rotations were obtained: $+10.7,+10.5,+8.5$, and +8.3 , after 4, 60, 1,500 and 3,000 minutes, respectively. Analysis calculated for $\mathrm{C}_{24} \mathrm{H}_{34} \mathrm{O}_{17}$ : C, 48.46; H, 5.77. Found: C, 48.50; H, 5.74.

A methoxyl determination by Zeisel's method did not give any silver iodide; hence the substance does not contain a methoxyl group. 
It gave essentially the same acetyl value when hydrolyzed with acids as with alkalies. The compound $(0.2698 \mathrm{~g})$ was refluxed with $50 \mathrm{ml}$ of $0.2 \mathrm{~N}$ hydrochloric acid. The quantity of acetic acid which was liberated was equivalent to $27.20 \mathrm{ml}$ of $0.1 \mathrm{~N}$ solution; the theoretical amount of acetic acid which could be derived from the sample or hexaacetyl-4-glucosido-mannose is $27.24 \mathrm{ml}$ of 0.1 solution. The alkaline hydrolysis was conducted in aqueous acetone solution. The sample $(0.2330 \mathrm{~g})$ was dissolved in about $10 \mathrm{ml}$ of acetone and $30 \mathrm{ml}$ of $0.1 \mathrm{~N}$ $\mathrm{NaOH}$ added. The solution was allowed to stand about two hours at room temperature. It was then titrated with standard acid. The titration showed that $23.33 \mathrm{ml}$ of $0.1 \mathrm{~N}$ alkali had been neutralized; the theoretical amount of alkali which would be required for the saponification of six acetyl groups in the sample, is $23.5 \mathrm{ml}$ of $0.1 \mathrm{~N}$ solution.

On acetylation with acetic anhydride and pyridine, hexa-acetyl-4glucosido-mannose gives the previously known $\alpha$-octa-acetyl-4-glucosido-mannose. The sample $(0.5 \mathrm{~g})$ of hexa-acetyl-4-glucosidomannose was added to a cold $\left(-10^{\circ} \mathrm{C}\right.$.) mixture of acetic anhydride $(7.5 \mathrm{ml})$ and pyridine $(5 \mathrm{ml})$. The mixture was shaken until solution was complete and allowed to stand 18 hours at $0^{\circ} \mathrm{C}$. It was then poured into ice water, and the product separated in the customary manner. The yield of pure octa-acetyl-4-glucosido-mannose was 0.5 g. It melted at $203^{\circ}$ and gave $[\alpha]_{D}^{20}=+36.2$. These values are in agreement with properties of octa-acetyl-4-glucosido-mannose as reported by Brauns. ${ }^{30}$

\section{PREPARATION OF CHLORO-HEPTA-ACETYL-4-GLUCOSIDO-MANNOSE FROM HEPTA-ACETYL-4-GLUCOSIDO- $\gamma$-METHYL-MANNOSIDE}

Two grams of hepta-acetyl-4-glucosido- $\gamma$-methyl-mannoside was dissolved in $20 \mathrm{ml}$ of chloroform. The specific rotation was then -12.7 . Upon the addition of $15 \mathrm{ml}$ of chloroform containing $0.149 \mathrm{~g}$ of hydrogen chloride the specific rotation changed rapidly, becoming constant in six minutes, $[\alpha]_{\mathrm{D}}^{20}=+29.8$. After 15 minutes the solution was washed in a separatory funnel with ice water, followed by a cold dilute solution of sodium bicarbonate, and again with ice water. After evaporation of the dry chloroform solution chloro-hepta-acetyl4-glucosido-mannose was obtained in the crystalline state. The crystals were washed with dry ether and then recrystallized from ethyl acetate by the addition of petroleum ether. About $1.3 \mathrm{~g}$ of the product was obtained. The recrystallized substance melted at $168^{\circ}$ and gave $[\alpha]_{\mathrm{D}}^{20}=+50.4(0.4605 \mathrm{~g}$ dissolved in $20 \mathrm{ml}$ of chloroform read $6.7^{\circ} \mathrm{S}$. in a $2 \mathrm{dm}$ tube). According to Brauns chloro-heptaacetyl-4-glucosido-mannose melts at $172^{\circ}$ and gives $[\alpha]_{D}^{20}=+51.2$. Analysis calculated for $\mathrm{C}_{26} \mathrm{H}_{35} \mathrm{O}_{17} \mathrm{Cl}: \mathrm{Cl}, 5.42$. Found: $\mathrm{Cl}, 5.35$.

Chloro-hepta-acetyl-4-glucosido-mannose was also obtained by the use of titanium tetrachloride. A solution of $1.2 \mathrm{~g}$ of anhydrous titanium tetrachloride in $20 \mathrm{ml}$ of chloroform was added to $4 \mathrm{~g}$ of heptaacetyl-4-glucosido- $\gamma$-methyl-mannoside dissolved in $50 \mathrm{ml}$ of chloroform. After refluxing four hours the cold chloroform solution was successively washed with ice water, dilute sodium bicarbonate solution and ice water. It was then dried with sodium sulphate and finally evaporated in vacuo. About $3.8 \mathrm{~g}$ of crystalline product was obtained. After recrystallization from ethyl acetate it melted at

${ }^{30}$ Brauns, J. Am. Chem. Soc., vol. 48, p. 2784; 1926. 
$169^{\circ}$ and gave in chloroform solution $[\alpha]_{\mathrm{D}}^{20}=+50.1(0.6080 \mathrm{~g}$ of substance dissolved in $20 \mathrm{ml}$ of solution read $8.8^{\circ} \mathrm{S}$. in a $2 \mathrm{dm}$ tube). These properties indicate that the substance is chloro-hepta-acetyl-4glucosido-mannose. Analysis calculated for $\mathrm{C}_{26} \mathrm{H}_{35} \mathrm{O}_{17} \mathrm{Cl}: \mathrm{Cl}, 5.42$. Found: $\mathrm{Cl}, 5.29$.

\section{HEPTA-ACETYL-4-GLUCOSIDO-MANNOSE}

Two grams of bromo-hepta-acetyl-4-glucosido-mannose was added to $50 \mathrm{ml}$ of cold $\left(-10^{\circ} \mathrm{C}\right.$.) acetone containing $5 \mathrm{ml}$ of water and $5 \mathrm{~g}$ of silver carbonate. The mixture was shaken until all the bromine was converted into silver bromide, which was separated by filtration. The solution was allowed to evaporate spontaneously in an open beaker. Slender prismatic, needlelike crystals separated during the course of several hours. The crystals were collected upon a filter and washed with water. After drying at $50^{\circ}$ in racuo the substance weighed $1.5 \mathrm{~g}$ and melted at $105^{\circ}$ to $110^{\circ}$. After recrystallization from hot water, in which it is difficultly soluble, it melted at $110^{\circ}$. Although the new substance is very soluble in ethyl acetate, chloroform, ether, and alcohol, it does not crystallize well from these solvents.

The optical rotation was determined in chloroform, methyl alcohol, and pyridine solutions. The recrystallized compound gave in chloroform, $[\alpha]_{D}^{20}=+11.7$ (1.3183 $\mathrm{g}$ of the substance dissolved in $25 \mathrm{ml}$ read $3.56^{\circ} \mathrm{S}$. in a $2 \mathrm{dm}$ tube); in methyl alcohol, $[\alpha]_{\mathrm{D}}^{20}=+11.6$ $(0.4117 \mathrm{~g}$ of the substance dissolved in $25 \mathrm{ml}$, five minutes after solution, read $1.10^{\circ} \mathrm{S}$. in a $2 \mathrm{dm}$ tube). The optical rotations of the solutions just given were read at intervals during the day and no changes were detected. However, in pyridine solution a slow mutarotation was observed. The sample $(0.2776 \mathrm{~g})$ was dissolved in sufficient pyridine to give $10 \mathrm{ml}$ of solution and the optical rotation was followed in a $2 \mathrm{dm}$ tube with a Bates saccharimeter. $[\alpha]_{\mathrm{D}}^{20}=+4.0$, $+4.6,+5.9,+6.9,+9.0$, and +11.7 after $0.1,0.5,2.0,4.0,10.0$, and 60.0 hours, respectively; the rotation was virtually constant after 60 hours. Analysis calculated for $\mathrm{C}_{28} \mathrm{H}_{28} \mathrm{O}_{18}$ : C, $49.03 ; \mathrm{H}, 5.70$. Found: C, 48.70; H, 5.82.

A sample of the compound $(0.1770 \mathrm{~g})$ was refluxed four hours with $50 \mathrm{ml}$ of $0.25 \mathrm{~N}$ sulphuric acid. The back titration with standard alkali gave a value equivalent to an increase of $19.33 \mathrm{ml} 0.1 \mathrm{~N}$ acid; the theoretical quantity which could be derived from the sample of hepta-acetyl-4-glucosido-mannose is $19.47 \mathrm{ml} 0.1 \quad N$ acid. On acetylation with pyridine and acetic anhydride it gives $\alpha$-octa-acetyl4 -glucosido-mannose. The sample $(1.5 \mathrm{~g})$ of hepta-acetyl-4-glucosidomannose was dissolved in a cold $\left(-10^{\circ}\right)$ mixture of $10 \mathrm{ml}$ of pyridine and $10 \mathrm{ml}$ of acetic anhydride. After standing overnight in the refrigerator, the mixture was poured into ice water. The product which separated after recrystallization from methyl alcohol weighed $1.2 \mathrm{~g}$. The substance was $\alpha$-octa-acetyl-4-glucosido-mannose, $[\alpha]_{D}^{20}=+36$. The mother liquor gave $0.17 \mathrm{~g}$ of crude crystalline material which melted at $160^{\circ}$ and gave $[\alpha]_{D}^{20}=+1.0$. The fact that $\alpha$-octa-acetyl-4-glucosido-mannose is obtained in good yield on acetylation under the conditions just given indicates that the new compound is largely the alpha form of hepta-acetyl-4-glucosidomannose. The explanation for the slight mutarotation in pyridine from a less dextro to a more dextro rotation must await further experimental study. 


\section{4-GLUCOSIDO- $\beta$-MANNOSE}

Crystalline 4-glucosido- $\beta$-mannose was prepared for the first time and its mutarotation measured. Five grams of 4 -glucosido- $\alpha$ mannose monohydrate was dissolved in about $150 \mathrm{ml}$ of hot c. p. methyl alcohol. The mixture was warmed on the steam bath for about 30 minutes, and then it was allowed to cool slowly. On standing, crystallization occurred. Two types of crystals were observed. One of these is the anhydrous alpha form of the sugar and the other the anhydrous beta form. The alpha isomer separates in parallelepipeds which frequently become truncated, while the beta separates in slender needlelike prisms. The crude product was crystallized by dissolving it in a very small quantity of water and then adding cold glacial acetic acid. About $1.5 \mathrm{~g}$ of the nearly pure substance crystallized from the solution. The crude product, after thorough washing with warm methyl alcohol, was immersed in methyl alcohol and allowed to stand in a tightly stoppered flask. After several days the product was separated by filtration, washed with methyl alcohol, and dried at $60^{\circ} \mathrm{C}$. Although it did not melt very sharply, it appeared homogeneous, and subsequent preparations gave essentially the same properties. It melted at $203^{\circ}$ to $205^{\circ}$ with decomposition. Analysis calculated for $\mathrm{C}_{12} \mathrm{H}_{22} \mathrm{O}_{11}$ : C, 42.08; $\mathrm{H}, 6.48$. Found: $\mathrm{C}, 42.14 ; \mathrm{H}, 6.47$. The optical rotation was measured at $20^{\circ} \mathrm{C}$. A sample $(0.8673 \mathrm{~g})$ of the finely powdered 4 -glucosido- $\beta$-mannose was weighed into a glass-stoppered flask and $24.8857 \mathrm{~g}$ of water was added from a pipette which had been calibrated and found to deliver that weight of water in 20 second. The sugar was completely dissolved in 10 seconds after the addition of the first drop of water. Time was measured from the moment it was believed that half of the sugar was dissolved (three seconds after adding the first drop of water).

The following saccharimeter readings were observed using a $2 \mathrm{dm}$ water-jacketed tube: -1.04 (3 minutes); -0.98 (4 minutes), -0.88 (5 minutes), -0.43 (10 minutes), +0.12 (20 minutes), +0.97 (50 minutes), and +1.27 (equilibrium value). The density of the solution was 1.008. The following specific rotations are obtained from the figures: $[\alpha]_{\mathrm{D}}^{20}=-6.5$ (extrapolated value to zero time), -5.3 (3 minutes), -5.0 (4 minutes), -4.5 (5 minutes), -2.2 (10 minutes), +0.6 (20 minutes), +4.9 (50 minutes), and +6.5 (equilibrium value). The initial rotation $(-6.5)$ is of the same order as the value $[\alpha]_{\mathrm{D}}^{20}$ $=-1.0$ obtained by Haworth, Hirst, Streight, Thomas, and Webb ${ }^{31}$ by indirect means. These investigators were unable to obtain the crystalline beta compound, but estimated its rotation by the solubility method of Hudson and Yanovsky. ${ }^{32}$ The equilibrium rotation is in accord with the value $(+5.88)$ previously reported for the monohydrate; the rotation for the monohydrate is equivalent to $[\alpha]_{D}^{20}=+6.2$ for the anhydrous sugar. This value is considerably less than the value $(+12.5)$ reported by Haworth, Hirst, Streight, Thomas, and Wébb.

\footnotetext{
31 See footnote 7, p. 1116. $\quad 32$ Hudson and Yanovsky, J. Am. Chein. Soc., vol. 39, p. 1035; 1917.
} 


\section{9. $\beta$-OCTA-ACETYL-4-GLUCOSIDO-MANNOSE}

A finely powdered sample $(1.1 \mathrm{~g})$ of 4 -glucosido- $\beta$-mannose was shaken with a cold $\left(0^{\circ}\right)$ solution of $25 \mathrm{ml}$ of pyridine and $30 \mathrm{ml}$ of acetic anhydride. When solution was complete (eight hours) the reaction mixture was allowed to stand in the refrigerator. After two days the product was isolated by pouring the mixture into ice water and separating the crystalline material. The crude product weighed $1.85 \mathrm{~g}$, melted at $155^{\circ}$ to $160^{\circ}$, and gave $[\alpha]_{\mathrm{D}}^{20}=+6.8$. This product consisted of a mixture of $\alpha$ - and $\beta$-octa-acetyl-4-glucosido-mannose. The separation of the two substances by fractional crystallization from the usual solvents is very difficult. It was found, however, that the alpha form may be separated after about three crystallizations from toluene. The impure beta form, which crystallizes from the mother liquors on evaporation, may be purified by several crystallizations from ethyl acetate by the addition of ether. The product so obtained gave $[\alpha]_{D}^{20}=-11.8$; after one additional crystallization, $[\alpha]_{D}^{20}=-13.04$; after two crystallizations, $[\alpha]_{\mathrm{D}}^{20}=-12.96$. It melted sharply at $165^{\circ}$. Analysis calculated for $\mathrm{C}_{28} \mathrm{H}_{3 \mathrm{~S}} \mathrm{O}_{19}$ : C, 49.54; $\mathrm{H}, 5.65$. Found: C, 49.54; H, 5.65.

\section{ACKNOWLEDGMENT}

The writer expresses his appreciation to Miss Harriet Frush and other members of the polarimetry section of the Bureau of Standards, who have been of assistance in the course of the investigation.

Washington, July 30, 1931. 